\title{
Arrow's Impossibility Theorem
}

\author{
Freek Wiedijk \\ Institute for Computing and Information Sciences \\ Radboud University Nijmegen \\ Toernooiveld 1, 6525 ED Nijmegen, The Netherlands
}

Summary. A formalization of the first proof from [6].

MML identifier: ARROW, version: 7.8.05 4.87.985

The terminology and notation used here are introduced in the following articles: $[11],[13],[12],[10],[9],[5],[2],[3],[1],[8],[4]$, and [7].

\section{Preliminaries}

Let $A, B^{\prime}$ be non empty sets, let $B$ be a non empty subset of $B^{\prime}$, let $f$ be a function from $A$ into $B$, and let $x$ be an element of $A$. Then $f(x)$ is an element of $B$.

Next we state two propositions:

(1) For every finite set $A$ such that $\operatorname{card} A \geq 2$ and for every element $a$ of $A$ there exists an element $b$ of $A$ such that $b \neq a$.

(2) Let $A$ be a finite set. Suppose card $A \geq 3$. Let $a, b$ be elements of $A$. Then there exists an element $c$ of $A$ such that $c \neq a$ and $c \neq b$.

\section{Linear Preorders and Linear Orders}

In the sequel $A$ denotes a non empty set and $a, b, c$ denote elements of $A$.

Let us consider $A$. The functor LinPreorders $A$ is defined by the condition (Def. 1). 
(Def. 1) Let $R$ be a set. Then $R \in \operatorname{LinPreorders~} A$ if and only if the following conditions are satisfied:

(i) $R$ is a binary relation on $A$,

(ii) for all $a, b$ holds $\langle a, b\rangle \in R$ or $\langle b, a\rangle \in R$, and

(iii) for all $a, b, c$ such that $\langle a, b\rangle \in R$ and $\langle b, c\rangle \in R$ holds $\langle a, c\rangle \in R$.

Let us consider $A$. Note that LinPreorders $A$ is non empty.

Let us consider $A$. The functor LinOrders $A$ yielding a subset of LinPreorders $A$ is defined by:

(Def. 2) For every element $R$ of LinPreorders $A$ holds $R \in \operatorname{LinOrders~} A$ iff for all $a, b$ such that $\langle a, b\rangle \in R$ and $\langle b, a\rangle \in R$ holds $a=b$.

Let $A$ be a set. One can verify that there exists an order in $A$ which is connected.

Let us consider $A$. Then LinOrders $A$ can be characterized by the condition:

(Def. 3) For every set $R$ holds $R \in \operatorname{LinOrders} A$ iff $R$ is a connected order in $A$.

Let us consider $A$. One can verify that $\operatorname{LinOrders} A$ is non empty.

In the sequel $o, o^{\prime}$ are elements of LinPreorders $A$ and $o^{\prime \prime}$ is an element of LinOrders $A$.

Let us consider $A, o, a, b$. The predicate $a \leq_{o} b$ is defined by:

(Def. 4) $\langle a, b\rangle \in o$.

Let us consider $A, o, a, b$. We introduce $b \geq_{o} a$ as a synonym of $a \leq_{o} b$. We introduce $b<_{o} a$ as an antonym of $a \leq_{o} b$. We introduce $a>_{o} b$ as an antonym of $a \leq_{o} b$.

We now state a number of propositions:

(3) $a \leq_{o} a$.

(4) $a \leq_{o} b$ or $b \leq_{o} a$.

(5) If $a \leq_{o} b$ or $a<_{o} b$ and if $b \leq_{o} c$ or $b<_{o} c$, then $a \leq_{o} c$.

(6) If $a \leq_{o^{\prime \prime}} b$ and $b \leq_{o^{\prime \prime}} a$, then $a=b$.

(7) If $a \neq b$ and $b \neq c$ and $a \neq c$, then there exists $o$ such that $a<_{o} b$ and $b<_{o} c$.

(8) There exists $o$ such that for every $a$ such that $a \neq b$ holds $b<_{o} a$.

(9) There exists $o$ such that for every $a$ such that $a \neq b$ holds $a<_{o} b$.

(10) If $a \neq b$ and $a \neq c$, then there exists $o$ such that $a<_{o} b$ and $a<_{o} c$ and $b<_{o} c$ iff $b<_{o^{\prime}} c$ and $c<_{o} b$ iff $c<_{o^{\prime}} b$.

(11) If $a \neq b$ and $a \neq c$, then there exists $o$ such that $b<_{o} a$ and $c<_{o} a$ and $b<_{o} c$ iff $b<_{o^{\prime}} c$ and $c<_{o} b$ iff $c<_{o^{\prime}} b$.

(12) Let $o, o^{\prime}$ be elements of LinOrders $A$. Then $a<_{o} b$ iff $a<_{o^{\prime}} b$ and $b<_{o} a$ iff $b<_{o^{\prime}} a$ if and only if $a<_{o} b$ iff $a<_{o^{\prime}} b$.

(13) Let $o$ be an element of LinOrders $A$ and $o^{\prime}$ be an element of LinPreorders $A$. Then for all $a, b$ such that $a<_{o} b$ holds $a<_{o^{\prime}} b$ if and only 
if for all $a, b$ holds $a<_{o} b$ iff $a<_{o^{\prime}} b$.

\section{Arrow's Theorem}

For simplicity, we follow the rules: $A, N$ are finite non empty sets, $a, b$ are elements of $A, i, n$ are elements of $N, p, p^{\prime}$ are elements of (LinPreorders $\left.A\right)^{N}$, and $f$ is a function from (LinPreorders $A)^{N}$ into LinPreorders $A$.

We now state the proposition

(14) Suppose that

(i) for all $p, a, b$ such that for every $i$ holds $a<_{p(i)} b$ holds $a<_{f(p)} b$,

(ii) for all $p, p^{\prime}, a, b$ such that for every $i$ holds $a<_{p(i)} b$ iff $a<_{p^{\prime}(i)} b$ and $b<_{p(i)} a$ iff $b<_{p^{\prime}(i)} a$ holds $a<_{f(p)} b$ iff $a<_{f\left(p^{\prime}\right)} b$, and

(iii) $\quad$ card $A \geq 3$.

Then there exists $n$ such that for all $p, a, b$ such that $a<_{p(n)} b$ holds $a<_{f(p)} b$.

In the sequel $p, p^{\prime}$ denote elements of $(\operatorname{LinOrders} A)^{N}$ and $f$ denotes a function from (LinOrders $A)^{N}$ into LinPreorders $A$.

One can prove the following proposition

(15) Suppose that

(i) for all $p, a, b$ such that for every $i$ holds $a<_{p(i)} b$ holds $a<_{f(p)} b$,

(ii) for all $p, p^{\prime}, a, b$ such that for every $i$ holds $a<_{p(i)} b$ iff $a<_{p^{\prime}(i)} b$ holds $a<_{f(p)} b$ iff $a<_{f\left(p^{\prime}\right)} b$, and

(iii) $\quad$ card $A \geq 3$.

Then there exists $n$ such that for all $p, a, b$ holds $a<_{p(n)} b$ iff $a<_{f(p)} b$.

\section{REFERENCES}

[1] Grzegorz Bancerek. Cardinal numbers. Formalized Mathematics, 1(2):377-382, 1990.

[2] Czesław Byliński. Functions and their basic properties. Formalized Mathematics, 1(1):5565, 1990.

[3] Czesław Byliński. Functions from a set to a set. Formalized Mathematics, 1(1):153-164, 1990.

[4] Czesław Byliński. Some basic properties of sets. Formalized Mathematics, 1(1):47-53, 1990.

[5] Agata Darmochwał. Finite sets. Formalized Mathematics, 1(1):165-167, 1990.

[6] John Geanakoplos. Three brief proofs of arrow's impossibility theorem. Cowles Foundation Discussion Papers 1123R3, Cowles Foundation, Yale University, April 1996. Available at http://ideas.repec.org/p/cwl/cwldpp/1123r3.html.

[7] Andrzej Trybulec. Subsets of complex numbers. To appear in Formalized Mathematics.

[8] Andrzej Trybulec. Function domains and Frænkel operator. Formalized Mathematics, 1(3):495-500, 1990.

[9] Andrzej Trybulec. Tarski Grothendieck set theory. Formalized Mathematics, 1(1):9-11, 1990.

[10] Zinaida Trybulec. Properties of subsets. Formalized Mathematics, 1(1):67-71, 1990.

[11] Edmund Woronowicz. Relations and their basic properties. Formalized Mathematics, 1(1):73-83, 1990.

[12] Edmund Woronowicz. Relations defined on sets. Formalized Mathematics, 1(1):181-186, 1990. 
[13] Edmund Woronowicz and Anna Zalewska. Properties of binary relations. Formalized Mathematics, 1(1):85-89, 1990.

Received August 13, 2007 